\title{
Study on Spectrum Allocation and Optimization of Wireless Communication Networks Based on SFOA
}

\author{
Yu Zhang ${ }^{1,2}$ and Jianying Li $\mathbb{i D}^{1}$ \\ ${ }^{1}$ Northwestern Polytechnical University, Xi'an, 710072 Shaanxi, China \\ ${ }^{2}$ Guangdong Mechanical \& Electrical Polytechnic Guangzhou 510515, China \\ Correspondence should be addressed to Jianying Li; zyu_2015160528@mail.nwpu.edu.cn
}

Received 30 April 2021; Revised 26 July 2021; Accepted 5 August 2021; Published 25 September 2021

Academic Editor: Wenqing Wu

Copyright ( 2021 Yu Zhang and Jianying Li. This is an open access article distributed under the Creative Commons Attribution License, which permits unrestricted use, distribution, and reproduction in any medium, provided the original work is properly cited.

\begin{abstract}
Wireless communication network spectrum is a limited resource. With the rapid increase of mobile communication services in recent years, traditional spectrum allocation methods are only based on a fixed spectrum allocation strategy, which often results in uneven and wasteful resource allocation. Therefore, spectrum allocation and the optimization problem need to be solved urgently. The application of semantic mobile computing in the Internet of Things and the research of emerging bionic models provide new ideas for this problem. In order to solve the problem of spectrum optimization and allocation, this paper proposes an optimization algorithm that simulates fisherman fishing to reasonably arrange the allocation and optimization of wireless network spectrum. This paper selects SFOA and the other two algorithms, designs experimental functions to perform calculations separately, obtains relevant data indicators, and uses comparative analysis to analyze. The analysis shows that in terms of performance, the success rate of SFOA is higher than that of PSO, and the success rate of the two function calculations has reached $100 \%$. In the signal-to-noise ratio analysis, when the signal-to-noise ratio is $-4 \mathrm{~dB}$, the throughput of GPSO reaches the maximum value of 0.17 , the throughput of PSO reaches the maximum value of 0.56 , and the throughput of SFOA reaches 1 , which shows that SFOA is adopted. The stability and accuracy of the algorithm are higher than the other two algorithms, and in the case of high signal-to-noise ratio, the advantages of the SFOA algorithm are also more obvious. This shows that the use of this algorithm will be very helpful for spectrum allocation and optimization. Because SFOA has high stability and accuracy, through reasonable adjustment and improvement, it can make good use of spectrum allocation and optimization. Chinese wireless communication network and the development of Internet of Things technology are of great significance.
\end{abstract}

\section{Introduction}

Wireless communication uses electromagnetic wave signals to propagate and exchange information in space. It is not restricted by the geographical environment. When the application environment changes, wireless communication only needs slight adjustments to quickly adapt to the new environment. The wireless communication network system has been implemented in many countries. Using the radio frequency identification technology in the Internet of Things, combined with the reasonable allocation and optimization of the spectrum, can minimize interference to other users. Mobile computing refers to the existence of movable devices, users, or programs in a distributed system, and the corresponding computing mode needs to support the mobility of these entities; in a narrow sense, mobile computing refers to the following: users carry mobile devices to access information services through public facilities, regardless of their what physical location is it or what kind of movement behavior it has. Mobile computing technology has developed rapidly in recent years, and the technology has become quite mature. For smart phones, mobile technology not only provides rich content, but also has powerful functions that can be expanded infinitely and the upgradeability of hardware, which enables it to truly realize the integration of communications, computers, and the Internet. 
In this regard, many scholars at home and abroad have conducted a lot of related research. Bertini et al. believe that heuristic evaluation is very popular in desktop and web interfaces, whether it is used in actual design or as a research topic. Compared with full user research, heuristic evaluation may be cost-effective. Therefore, in the case of limited resource input, this evaluation can detect large usage defects before the resources are fully developed, and these defects may appear on both mobile computing and traditional interfaces. But usability evaluation will also bring some specific problems, involving screen space, user attention, and background factors. The authors introduced an improved heuristic method suitable for mobile computing evaluation in their research, which was systematically derived from a large amount of literature and verified by certain experience. These methods can provide a good foundation for heuristic evaluation in mobile computing. The study not only introduced the practical use of heuristic evaluation, but also analyzed its application prospects. However, the study lacked a large amount of relevant data, and its credibility was not strong [1]. Ma et al. introduced that their research optimizes power and spectrum allocation at the same time to improve the communication quality of the demand side in the smart grid, thereby further reducing the cost of utility companies. First, the authors model the electricity bill based on the adjustment error caused by the direct load control in the smart grid. Then, according to the confidence level of the frequency band and the highest cost of the utility company, the subbands are allocated to different data aggregator units. An algorithm is designed to optimize the transmission power of the repeater and optimize the spectrum allocation to reduce the cost of the utility company. The simulation results show that the packet loss rate and cost of public utility companies can be greatly reduced. This research compares with other solutions by designing its own scheme, and the consideration is more comprehensive, but there is a lack of discussion on how to realize spectrum data sharing $[2,3]$. Sohul et al. believe that wireless communication plays a vital role in national security and disaster relief and is widely used by emergency personnel such as police officers, firefighters, and ambulances. In an emergency, the first discoverer of the accident must always keep in touch with the remote service center for effective cooperation and coordination. However, in many emergency situations, the existing public safety services cannot meet the needs of users, which usually results in excessive traffic load. Therefore, increasing network capacity is one of the main problems at present, and spectrum sharing is a key solution. Through the spectrum sharing partnership between public safety service organizations and commercial networks, users can access the licensed public safety spectrum when needed to realize data sharing. In the study, the authors introduced different measures taken for the next generation of public safety networks. The authors also have an outlook on public safety systems that can be quickly deployed and reconfigured to meet the demand for critical communication infrastructure and discuss the value of spectrum sharing. In their research, the authors discussed the value of spectrum sharing in wireless communication and gave a very detailed introduction to its application. However, they failed to design a specific model for simulation experiments. It is difficult to predict the problems and problems that may occur in practical applications $[4,5]$.

This article is aimed at studying the optimization and allocation of wireless network communication spectrum. First, it summarizes the research results of some scholars at home and abroad and then introduces semantic mobile computing and its working principles, the basic principles of the SFOA algorithm model, and spectrum allocation and optimization. And comb and then design an experiment based on SFOA, compare with other algorithms, conduct indepth analysis of performance, operating speed, throughput, system benefits, etc., and then analyze the practical application of mobile computing in the Internet of Things. Finally, a brief summary of this article is made, and the shortcomings of this article are put forward. The innovation of this paper is to combine the current new bionic method SFOA algorithm for spectrum allocation and optimization analysis and design an experimental model that simulates fisherman fishing, which has profound significance for the reasonable utilization of wireless network spectrum resources in reality.

\section{Research Method of Spectrum Allocation and Optimization of Wireless Communication Network Based on SFOA}

2.1. Semantic Mobile Computing. Mobile computing is a new technology that has emerged with the development of honeycomb technology, wireless WLAN, wireless Internet, and GPS positioning technology. Mobile computing enables mobile terminal equipment to achieve network access, resource transmission, and information sharing in a wireless network environment. Its role is to provide customers with accurate, useful and immediate information anywhere, at any time, which has made a huge difference to people's lives [1]. Mobile computing is an extension of distributed computing, enabling computers or other devices to transmit data without a fixed physical device connected and to transmit this information to users in need. In this mode, users use portable computing devices to access information networks through wireless communication interfaces without being affected by changes in geographic locations $[6,7]$.

Mobile computing technology is to implement a mobile system for transaction and data processing in an environment with limited resources, intermittent connections, and lightweight computing. Due to the power limit of mobile devices, network instability caused by frequent movement, poor network performance, etc., frequent network disconnections occur under mobile computing. Therefore, it is necessary to maintain a normal user environment with intermittent connections. Critical transactions will not be undone due to disconnection [8]. The communication bandwidth, battery power, storage capacity, and processing capabilities of mobile devices are all limited. These restrictions require applications in mobile computing systems to use less memory, improve operating efficiency, and comprehensively use caching, multithreading, and other technologies to optimize processing. 
Process and improve efficiency in query processing, transaction management, storage management, etc.

2.1.1. Mobile Computing Working Mode. The working mode of mobile computing is shown in Figure 1, which includes three types of computing units: a mobile support node (MSS) with a wireless communication module, communicating with mobile devices, a fixed host (MS) without a wireless communication module, and a mobile terminal (MC). The mobile terminal is linked with the mobile support node core through the wireless network and finally enters the fixed network. Relative to the low credibility of the wireless network unit, the fixed network is called the credible part. In this working mode, MC can access a fixed network or other MC from any wireless network via MSS and enables MC to move freely in the wireless network while still maintaining the network connection between MC and MSS [9, $10]$

2.1.2. Semantic Cache. Mobile computing involves the problem of semantic caching. Cache technology has been applied in many fields. Caching technology has different meanings in different fields. In a traditional computing environment, caching technology refers to storing some commonly used data on high-speed devices, thereby improving processing performance. The caching technology in the mobile computing environment saves part of the server's data on the mobile client, thereby reducing network traffic [11]. Although their meanings are not exactly the same, their purpose is to improve data access performance, and mobile devices can effectively increase the cache hit rate by selecting a suitable cache replacement algorithm, thereby further improving computing efficiency.

Cache is divided into traditional cache and semantic cache. The comparison between the two is shown in Table 1. The traditional cache has low cache efficiency, high network cost, and low data processing efficiency. The cache mode used is generally divided into a page cache mode and a tuple cache mode. The semantic cache is a cache mode established based on the semantic correlation between customer query sentences. The query results are produced by mining the semantic information in the query words, and the query results and description information are cached to make the cache utilization more effective. The semantic cache first decomposes the query through the local system and then performs the trial query and the remaining query to obtain the result, and then, the server processes and exports and finally obtains the final result [12].

2.2. Description of Fisherman's Fishing Method SFOA. SFOA, also known as the optimization algorithm for simulating fisherman fishing, is a new type of bionic algorithm. The algorithm is simple in principle, involves fewer parameters, and is easy to operate. It provides a new idea for solving the allocation and optimization of the wireless communication network spectrum. How much fisherman fishes each time depends on where he casts the net, and the choice of where to cast the net is determined by the density of the fish [11]. This algorithm is a search method that simulates the fishing behavior of a fisherman based on the fishing behavior of the fisherman. Figure 2 is a real scene of the fisherman fishing.

The fishermen's fishing habits are as follows: initially, each fisherman randomly chooses a location to fish; the fisherman always randomly casts a fishing net around his location to try to understand the density of the fish around him and move within the maximum radius of the net, each time moving to a place with greater fish density than its current location; the fisherman always reports the fish density of his location to other teammates in a timely manner; when there is no better within the radius of the net, when the fisherman shrinks the net radius in order to accurately obtain the point with a higher density of fish nearby, and when the fisherman cannot find a better density point in the area of his maximum casting net radius, he will leave the current casting area and look for other casting points [13].

2.3. Wireless Communication. Wireless communication gets rid of the shackles of fixed location of wired communication equipment, can communicate anytime and anywhere, and is favored by users. At the same time, the continuous emergence of wireless communication services has also promoted the progress of wireless communication technology. With the continuous emergence of new technologies and the continuous expansion of wireless applications, the demand for wireless spectrum resources in communication systems is growing rapidly [14]. However, the available wireless spectrum resources are very limited, and the available wireless spectrum resources are increasingly scarce, which seriously hinders the development of communication technology. Therefore, allocation and optimization issues are very important.

2.3.1. Spectrum Allocation Problem. The problem of spectrum allocation in wireless communication networks is to effectively reuse frequency resources for communication nodes or communication links and reduce network interference. The problem of spectrum resource allocation stems from the mobile phone network. The carrier frequency is effectively allocated to the base station to avoid various interference. Therefore, the problem of spectrum allocation is also called the problem of frequency allocation $[15,16]$.

From the point of view of the allocation mode, the spectrum allocation technology can be divided into static spectrum allocation and dynamic spectrum allocation. Static spectrum allocation assigns fixed spectrum resources to specific users, and users cannot flexibly adjust according to their own needs. The advantage of this technology is that the allocation strategy is simple, but the spectrum utilization rate is low $[17,18]$. The dynamic spectrum allocation technology dynamically schedules spectrum resources among users according to network requirements, which can effectively meet the spectrum needs of different users, improve network capacity and spectrum utilization, and achieve better user performance experience. According to the spectrum access method, it can generally be divided into fully restricted spectrum allocation and partial restricted spectrum allocation; according to the network structure 


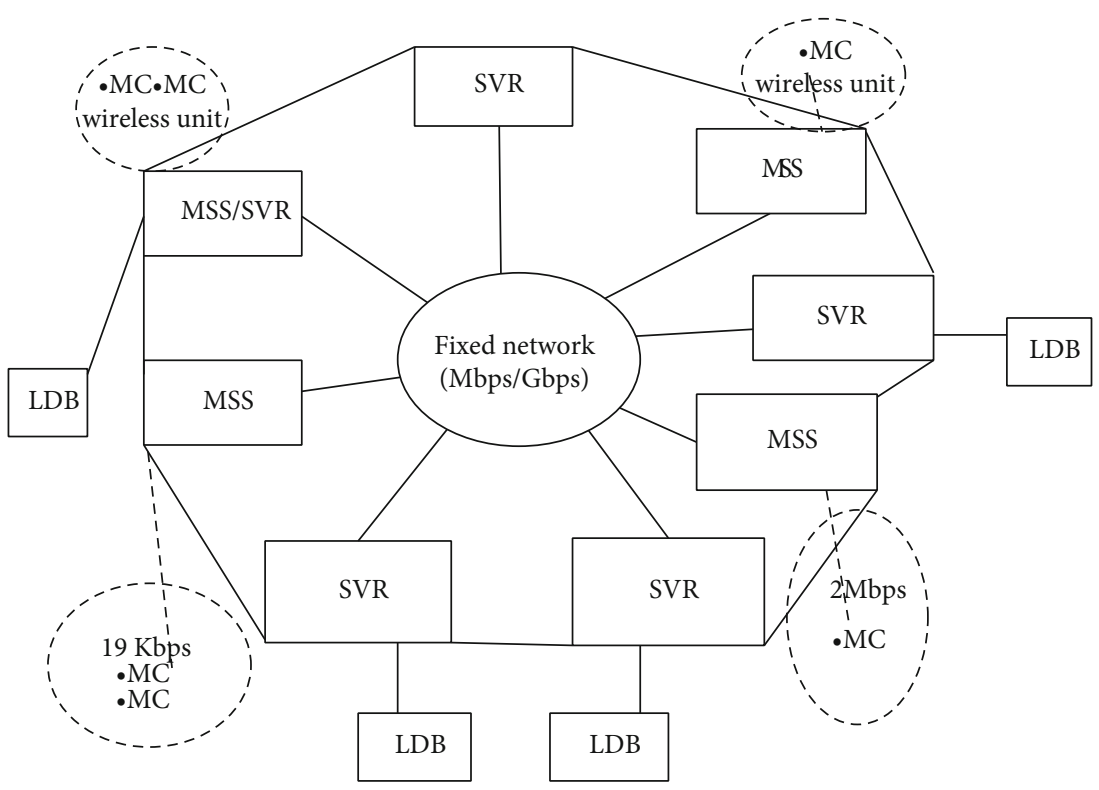

Figure 1: Model of mobile computing.

TABLE 1: Comparison between traditional cache and semantic cache.

\begin{tabular}{lccc}
\hline Index & Traditional cache & Semantic cache & Description \\
\hline Cache efficiency & Low & High & Contains historical query results with cache items as the granularity \\
Network cost & High & Low & Only transfer the remaining query data \\
Query processing efficiency & Low & High & Able to process in parallel \\
Adaptability & Bad & Good & More query results can be obtained in a disconnected environment \\
\hline
\end{tabular}

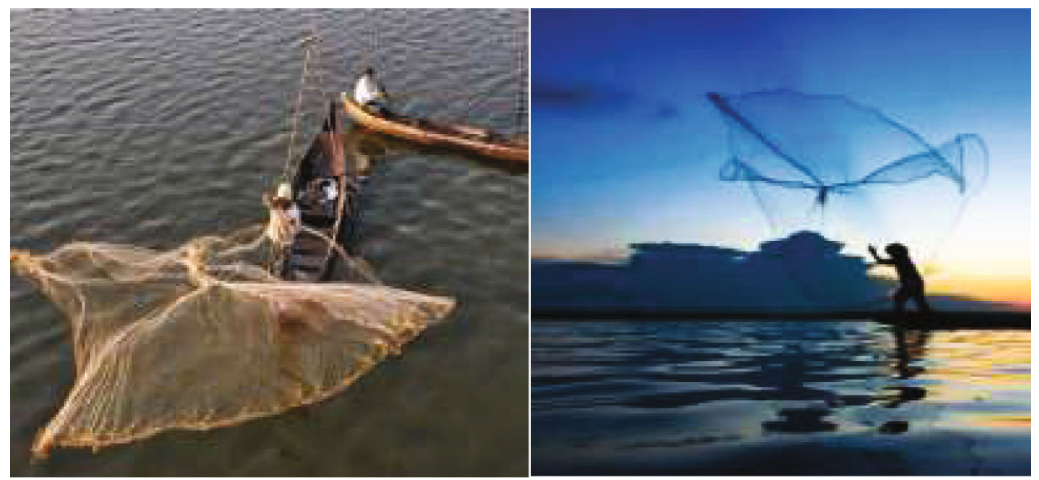

Figure 2: Fisherman fishing sketches (pictures from Baidu Picture).

classification, it can generally be divided into centralized spectrum allocation and distributed spectrum allocation; classified by cooperation method, it generally can be divided into for cooperative spectrum allocation and noncooperative spectrum allocation [19].

The principles of the spectrum allocation algorithm are as follows: to ensure the flexibility of the algorithm, and the time complexity should be low; to improve the overall performance of the network system, such as increasing the network capacity, reducing the network load, etc., and reducing the network signaling overhead (referring to the number of communication handshake) and computational complexity; must consider the application requirements in the system, network structure characteristics, cooperation forms, and other issues [20].

In the distribution, some interference problems will appear, mainly caused by stations working on the same channel or adjacent channels. Generally speaking, cochannel interference is the main source of interference and a key issue in spectrum allocation, while adjacent channel interference is the interference caused by modulated radio frequency signals entering adjacent channels. At present, this distribution technology has been applied in mobile cellular networks, wireless local area networks, and satellite 


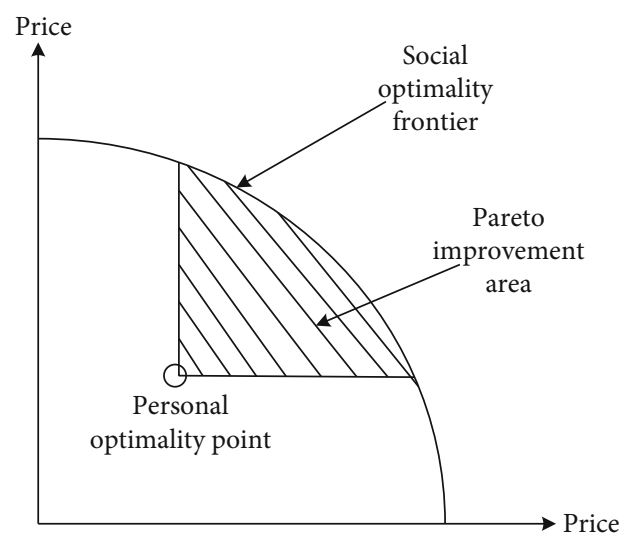

Figure 3: Relation between social and personal optimality.

communication networks. According to the different frequency allocation methods, similar channel allocation principles are adopted in the above applications, and one or more frequency points are allocated to the station to meet the communication needs and reduce network interference [21, 22].

On the issue of spectrum allocation, graph theory coloring models, auction bidding models, and interference temperature models are generally used. Here is an introduction to the interference temperature model. This model is used to manage and quantify the interference between wireless communication users. The main content is to monitor the power of the receiving end of the communication process. When using this model, the CU device operating in the licensed frequency band can monitor the interference situation at its location in real time and select the spectrum and configure the transmit power according to the interference situation [23]. The interference temperature threshold $T_{i}$ refers to the maximum value of interference that can be tolerated in a certain frequency band in a certain area. The interference temperature is defined as follows:

$$
T_{i}\left(f_{c}, A\right)=\frac{M_{i}\left(f_{c}, A\right)}{k A} .
$$

Among them, $f_{c}$ is the frequency center of the spectrum, $A$ is the bandwidth of the spectrum, $T_{i}$ is the noise temperature, and $k$ is the Boltzmann constant [24].

2.3.2. Spectrum Optimization Problem. For the spectrum optimization problem, game theory is mainly used. Game theory is a series of models and analysis tools used to study the interactive decision-making process of decision makers with contradictory goals, including the set of participants, the behavior of each participant, the preferences of the participants, the order of the game, and the benefits of the participants [25].

In the game, $K$ is often used to represent a game. The income of participant $z$ is represented by $P_{z}$, and $P_{z 1}$ is a multivariate function about the strategies of each participant. Therefore, the game $K$ with $n$ participants can be expressed as

$$
K=\left\{T_{1}, \cdots, T_{n} ; P_{1}, \cdots, P_{n}\right\}
$$

Among them, the strategy combination is

$$
t^{*}=\left(t_{1}^{*}, \cdots, t_{n}^{*}\right)
$$

If for any strategy $t_{i} \in T_{i}$, we have

$$
P_{z}\left(t^{*}\right) \geq P_{z}\left(t_{1}^{*}, \cdots, t_{z-1}^{*}, t_{z}, t_{z+1}^{*}, \cdots, t_{n}^{*}\right)
$$

It is said that the strategy combination is a Nash equilibrium of game $K$, which is called individual optimal. The combination of strategies in this state is stable. In addition, there is also a social optimum. As shown in Figure 3, it shows the relationship between personal optimal and social optimal [26].

\section{SFOA-Based Wireless Communication Network Allocation and Optimization Experiment}

3.1. Experiment Object. This experiment selects three algorithms of SFOA, PSO, and GPSO for comparative analysis. The SFOA aspect has been introduced before, so I will not repeat it here. PSO is also a kind of bionic algorithm model. Its principle is that each bird in the flock will constantly compare its own position and then adjust its speed and position through the bird closest to the food in the flock, thereby searching for food. GPSO, also known as gradient particle swarm optimization algorithm, has the shortcomings of slow convergence speed and easy to fall into local optimum [27, 28].

3.2. SFOA Experimental Procedure. The specific content of the algorithm is as follows:

Suppose a finite closed area is

$$
M=M_{1} \times M_{2} \times \cdots \times M_{k}
$$

The fisherman's status is

$$
Y=\left(y_{1}, y_{2}, \cdots, y_{k}\right) \in M
$$

$f(y)$ is the continuous objective function on $M$, that is, the density distribution function of the fish.

First, there are $x$ fishermen randomly distributed in $M$, and the initial position of fisherman $a$ is

$$
S_{0}^{(a)}=\left(y_{01}^{(a)}, y_{02}^{(a)}, \cdots, y_{0 n}^{(a)}\right)
$$

The fisherman $a$ casts the fishnets on the four sides of the initial position $S_{0}^{(a)}$, namely, up and down, front, back, left, and right and then gets the fishnet point set with the point $S_{0}^{(a)}$ as the center:

$$
\begin{aligned}
\beta_{0}^{(a)} & =\left\{Y^{(a)}=\left(w_{1}^{(a)}, w_{2}^{(a)}, \cdots, w_{n}^{(a)}\right) \mid w_{j}^{(a)}\right. \\
& \left.\in\left\{y_{0 j}^{(a)}-l_{j}^{(-)}, y_{0 j}^{(a)}, y_{0 j}^{(a)}+l_{j}^{(+)}\right\}, j=1,2, \cdots, n\right\} .
\end{aligned}
$$


When $y_{0 j}^{(a)}=d_{j}$, we have $l_{j}^{(-)}=0$, and $l_{j}^{(+)}<f_{j}-y_{o j}^{(a)}$. When $y_{0 j}^{(a)}=f_{j}$, we get $l_{j}^{(-)}<y_{0 j}^{(a)}-d_{j}$. And when $y_{0 j}^{(a)} \neq d_{j}$ and $y_{0 j}^{(a)} \neq f_{j}$, we get $l_{j}^{(-)}<y_{0 j}^{(a)}-d_{j}$ and $l_{j}^{(+)}<f_{j}-y_{o j}^{(a)}$, where $a=1,2, \cdots, k$ and $j=1,2, \cdots, n$.

In this area, there are three search methods, divided into mobile search, narrow search, and accelerated search.

3.2.1. Mobile Search. If the relationship between the initial position and the new position is

$f\left(Y_{0}^{(a)}\right)=\max _{Y^{(a)} \in \beta_{0}^{(a)}} f\left(Y^{(a)}\right)>f\left(S_{0}^{(i)}\right), Y_{0}^{(a)} \neq S_{0}^{(a)}, a=1,2, \cdots, k$.

Then, fisherman $a$ moves from the current location to the new location

$$
S_{1}^{(a)}=Y_{0}^{(a)}
$$

And repeat the previous method with $S_{1}^{(a)}$ as the new center in order to search for point $S_{2}^{(a)}$ where the density of fish is greater than that of point $S_{1}^{(a)}$. After a limited number of movements, the position of fisherman $a$ is $S_{0}^{(a)}, S_{1}^{(a)}, S_{2}^{(a)}$, $\cdots, S_{n}^{(a)}$.

3.2.2. Shrink Search. Assuming that after a finite number of state changes, the position of fisherman $a$ is

$$
S_{t}^{(a)}=\left(y_{t 1}^{(a)}, y_{t 2}^{(a)}, \cdots, y_{t n}^{(a)}\right)
$$

If the initial position and the new position meet,

$$
\max _{Y^{(a)} \in \beta_{0}^{(a)}} f\left(Y^{(a)}\right) \leq f\left(S_{0}^{(i)}\right) .
$$

Among them, we have

$$
\begin{aligned}
\beta_{t}^{(a)}= & \left\{Y_{t}^{(a)}=\left(w_{1}^{(a)}, w_{2}^{(a)}, \cdots, w_{n}^{(a)}\right) \mid w_{j}^{(a)}\right. \\
\in & \left.\left\{y_{t j}^{(a)}-l_{j}^{(-)}, y_{t j}^{(a)}, y_{t j}^{(a)}+l_{j}^{(+)}\right\}, j=1,2, \cdots, n\right\}, \\
& \quad a=1,2, \cdots, k .
\end{aligned}
$$

Then, the current position of fisherman $a$ is still $S_{t}^{(a)}$, and the net is cast once again around the current position $S_{t}^{(a)}$. At this time, another fishnet point set that is centered at $S_{t}^{(a)}$ is

$$
\begin{aligned}
\beta_{t+1}^{(a)} & =\left\{Y_{t+1}{ }^{(a)}=\left(w_{1}^{(a)}, w_{2}^{(a)}, \cdots, w_{n}^{(a)}\right) \mid w_{j}^{(a)}\right. \\
& \left.\in\left\{y_{t j}^{(a)}-l_{j}^{(-)}, y_{t j}^{(a)}, y_{t j}^{(a)}+l_{j}^{(+)}\right\}, j=1,2, \cdots, n\right\} .
\end{aligned}
$$

Among them, $l_{j}^{(-)}=\lambda l_{j}^{(-)}, l_{j}^{(+)}=\lambda l_{j}^{(+)}, 0<\lambda<1$, and $\lambda$ is the shrinkage coefficient.
3.2.3. Speedup Search. Suppose that after a limited number of position changes, the position of fisherman a remains unchanged, and

$$
\begin{aligned}
& f\left(S_{t}^{(a)}\right)-\max \left\{f(Y) \mid Y \in \beta_{t}^{(a)}, Y \neq S_{t}^{(a)}\right\}<e, \\
& f\left(S_{t}^{(a)}\right)<\max \left\{f\left(S_{t}^{(a)}\right), a=1,2, \cdots, k\right\}, \\
& \beta_{t}^{(a)}=\left\{Y_{t}^{(a)}=\left(w_{1}^{(a)}, w_{2}^{(a)}, \cdots, w_{n}^{(a)}\right) \mid w_{j}^{(a)}\right. \\
& \left.\in\left\{y_{t j}^{(a)}-l_{j}^{(-)}, y_{t j}^{(a)}, y_{t j}^{(a)}+l_{j}^{(+)}\right\}, j=1,2, \cdots, n\right\}, \\
& a=1,2, \cdots, k .
\end{aligned}
$$

Among them, $e>0$ is a self-defined smaller constant, and then, fisherman $a$ accelerates to move out of the local fishing area, so as not to fall into the local optimum.

3.2.4. Experimental Function. This experiment selects three functions $f_{1}, f_{2}$, and $f_{3}$ and uses the above three algorithms to calculate and analyze various experimental indicators: optimal solution, worst solution, average solution, standard deviation, number of successes, and average time-consuming. The optimal solution refers to the best situation that can achieve the goal, and the worst solution is the opposite. The average solution represents the general case. The standard deviation is used to detect the degree of distribution. The number of successes is used to indicate the accuracy and accuracy of the algorithm. The time is used to measure the running speed.

(1) $f_{1}(x)=\left(x_{1}{ }^{2}-x_{2}{ }^{2}\right)^{0.89}\left[\tan \left(x_{1}^{4} / x_{2}-5 x_{1}^{2}+0.5\right)\right],-50 \leq$ $x_{1} \leq 50,-50 \leq x_{2} \leq 50$

(2) $\left.f_{2}(x)=\sqrt{\left(x_{1} x_{2}-x_{1}^{2}\right)}\left(\cos x_{1} / 5 x_{2}-100 x_{1}\right)\right],-50 \leq x_{1}$ $\leq 50,-50 \leq x_{2} \leq 50$

(3) $f_{3}(x)=\sin \left[2 x_{2}{ }^{5}-5\left(x_{1}-7 x_{1}^{9}\right)\right]-3 \sqrt{x_{1}^{2}+x_{1}^{2}},-50$ $\leq x_{1} \leq 50,-50 \leq x_{2} \leq 50$

\section{SFOA-Based Wireless Communication Network Spectrum Allocation and Optimization Research Analysis}

4.1. SFOA and Other Algorithm Performance Analysis. In order to compare the performance of SFOA and other algorithms, this paper selects five indicators for evaluation and analysis: optimal solution, worst solution, average time-consuming, average solution, and success rate. In order to reduce the influence of randomness on the results of this experiment and make the comparison more reasonable, each algorithm must be run 100 times independently. The experimental data are recorded in Tables 2 and 3, and the running time is recorded in Table 4.

As shown in Table 2, SFOA and PSO algorithms are used to calculate the function $f_{1}$. The worst solution and optimal solution of the two are relatively close, but from the perspective of operating speed, the average time of SFOA is shorter, and its operating speed is higher than that of PSO. Both methods have a $100 \%$ operation success rate, which can 
TABLE 2: Experimental comparison results.

\begin{tabular}{lccc}
\hline & Function & SFOA & PSO \\
\hline \multirow{3}{*}{ Worst solution } & $f_{1}$ & $3.6738839402812837 e-16$ & $4.5987453450717516 e-03$ \\
& $x_{1}$ & 1.0082782276343545 & 1.0000027638792636 \\
& $x_{2}$ & 1.0097487234612278 & 1.0000076437264872 \\
Best solution & $f_{1}$ & $1.2637632476348764 e-07$ & $2.3784792384892744 e-12$ \\
& $x_{1}$ & 1.0000063272636327 & 0.9999999999993272 \\
Average time & $x_{2}$ & 1.0000083742637863 & 0.9999999999993627 \\
Average value & $f_{1}$ & $1.6372632636371863 e-09$ & 2.3787264378648972 \\
Success rate & $f_{1}$ & 1.836278 & 1.361728 \\
\hline
\end{tabular}

TABLE 3: Experimental comparison results.

\begin{tabular}{lccc}
\hline & Function & SFOA & PSO \\
\hline & $f_{2}$ & $6.6376246328796438 e-02$ & 4.0037678263879126 \\
Worst solution & $x_{1}$ & 1.3762986398723898 & 1.0000000007436482 \\
& $x_{2}$ & 1.7233823829083482 & -12.3727647836478386 \\
& $f_{2}$ & $1.2578647865874654 e-07$ & $2.0093487724893274 e-11$ \\
Best solution & $x_{1}$ & -6.2637263782638213 & 8.9274389372489327 \\
Average time & $x_{2}$ & 1.2743894598375758 & 3.3627361726334232 \\
Average value & $f_{2}$ & $4.3762863872687326 e-07$ & $4.8327928798217388 e-02$ \\
Success rate & $f_{2}$ & 1.293774 & 10.7483974 \\
\hline
\end{tabular}

Table 4: Performance contrast between two algorithms.

\begin{tabular}{lcccc}
\hline Function & Algorithm & Average value of function & Success times & Average working time \\
\hline \multirow{2}{*}{$f_{1}$} & SFOA & $3.473826292748262 e-022$ & 100 & 4.123 \\
& PSO & $4.382749823729834 e-012$ & 100 & 4.334 \\
$f_{2}$ & SFOA & $1.124683629426498 e-018$ & 100 & 0.892 \\
& PSO & $1.578497598437894 e-015$ & 100 & 1.783 \\
$f_{3}$ & SFOA & $9.000000000033443 e-002$ & 100 & 0.829 \\
& PSO & $9.000000472867823 e-001$ & 100 & 2.412 \\
\hline
\end{tabular}

indicate that the two algorithms have strong stability when calculating the function. Therefore, in order to avoid randomness, these two methods are used to calculate the function $f_{2}$.

It can be seen from Table 3 that the SFOA algorithm is generally better than the PSO algorithm. From the perspective of the optimal solution and the worst solution, the stability of SFOA is better than that of PSO. The success rate of SFOA algorithm operation is $100 \%$, while PSO is only $90 \%$. It can be seen that SFOA is not easy to fall into local extreme values, and its accuracy and stability are both better than PSO, and the retrieval quality is also the highest.

From the perspective of running time, as shown in Table 4 , when calculating functions $f_{1}, f_{2}$, and $f_{3}$, the average time used by SFOA is lower than PSO, and the number of runs in this experiment reaches 100 times, which can effec- tively avoid chance and randomness. It can be seen that the average operating speed of SFOA is higher than that of PSO, and the convergence speed of SFOA is faster than that of PSO. It can be seen from the above that the SFOA algorithm is generally better than the PSO algorithm in terms of optimization accuracy.

4.2. System Throughput and Signal-to-Noise Ratio Change Analysis. The signal-to-noise ratio is an important technical indicator used to measure the reliability of the communication quality of a communication system. It is often used to detect the number of information bits correctly transmitted by a system in a unit time, that is, the value of the throughput. This experiment limits the signal-to-noise ratio to between -16 and $4 \mathrm{~dB}$ and compares the system throughput of the three algorithms with different signal-to-noise ratios. 


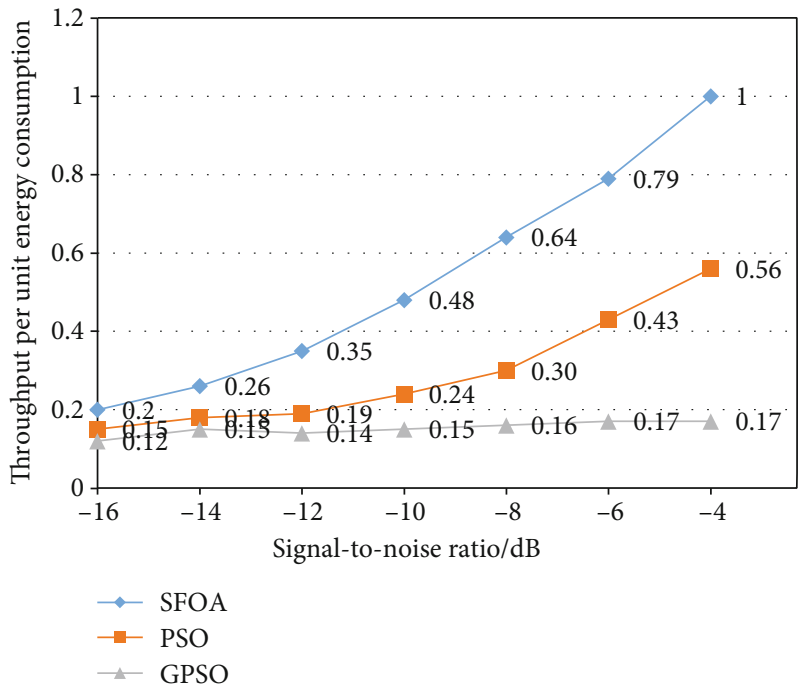

FIGURE 4: The relationship between normal throughput energy ratio and signal-to-noise ratio.

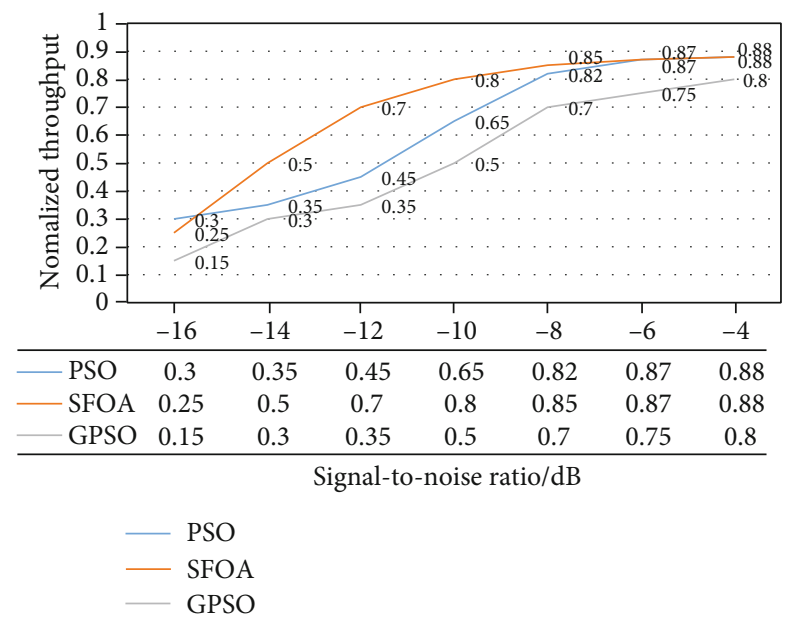

FIGURE 5: The relationship between throughput and signal-to-noise ratio under different algorithms.

As shown in Figure 4, in the range of signal-to-noise ratio of -16 to 4 , the throughput per unit energy consumption of SFOA is significantly higher than the throughput of the other two algorithms. Among them, the throughput of PSO is moderate, and the throughput of GPSO is the lowest. When the signal-to-noise ratio reaches $-4 \mathrm{~dB}$, the throughput of GPSO reaches the maximum of 0.17 , the throughput of PSO reaches the maximum of 0.56 , and the throughput of SFOA reaches 1 . It can be seen that the SFOA algorithm is significantly better than the other two algorithms.

As shown in Figure 5, it can be seen that under different signal-to-noise ratios, different algorithms have different throughputs. When the signal-to-noise ratio is $-16 \mathrm{~dB}$, the throughput of PSO is the highest at 0.3 , while the throughput of GPSO is the lowest at 0.15 , and when the signal-tonoise ratio is -14, the algorithm with the highest throughput is SFOA, which is 0.5 . When the signal-to-noise ratio is $-4 \mathrm{~dB}$, the throughput of PSO and SFOA is the highest, both being 0.88 . It can be seen that in the case of low signal-tonoise ratio, the throughput of the PSO algorithm is the best. When the signal-to-noise ratio is higher than $-8 \mathrm{~dB}$, the throughput of PSO and SFOA is close, while the throughput of GPSO is always worse than the two algorithms.

4.3. Changes in the Number of Users. Assuming that the user needs to continuously send information to its neighboring nodes at every moment, the user may be allocated multiple channels, and a certain channel may also be allocated to multiple conflict-free users. Therefore, this paper also analyzes the changes in the allocation performance of the spectrum under different algorithms with the increase of different users.

Figure 6(a) shows the situation when the number of primary users increases, and Figure 6(b) shows the situation when the number of cognitive users increases. It can be seen that as the number of users increases, the throughput decreases, that is, the spectrum allocation performance gradually decreases. But at the same time, it can be seen that as the number of users increases, the advantages between different algorithms are gradually becoming apparent. When the number of primary users increases, the advantages of SFOA and PSO algorithms gradually become greater than GPSO. When the number of cognitive users increases, the advantage of the SFOA algorithm is significantly higher than the other two algorithms at first, but when the number of cognitive users reaches 60, the advantage of the PSO algorithm begins to increase again.

As shown in Figure 7, when the number of users changes, the system benefits will also change. As the number of users increases, various spectrums among users will compete with each other, thereby increasing the interference effect on system benefits. It can be seen that the three algorithms will all be affected by the interference frequency after the number of users increases. Among them, the SFOA decreases the most slowly, and even when the number of users reaches 40 , the system revenue rebounds. The performance of the system revenue brought by the GPSO algorithm first decreases, then increases, and finally begins to decrease all the time. PSO maintains the same downward trend as SFOA, but the decline is higher than that of the SFOA algorithm.

The SFOA algorithm, as shown in Table 5, can always get a better optimal solution than the other two algorithms. In terms of average net loss, the SFOA algorithm has the lowest loss of 0.1248 , while the GPSO algorithm has a loss of 0.1294. In terms of standard deviation, SFOA is only 0.0019 , indicating that the algorithm is more accurate.

4.4. Semantic Mobile Computing in the Internet of Things Application Analysis. The application scenario of semantic mobile computing in the Internet of Things is for mobile RFID terminals, collecting data in a wireless network communication environment, and transmitting tags to the server through wireless communication. During the use of the mobile terminal, the collected information can be processed and transmitted at any time according to the change of the network communication access point, so that real-time 

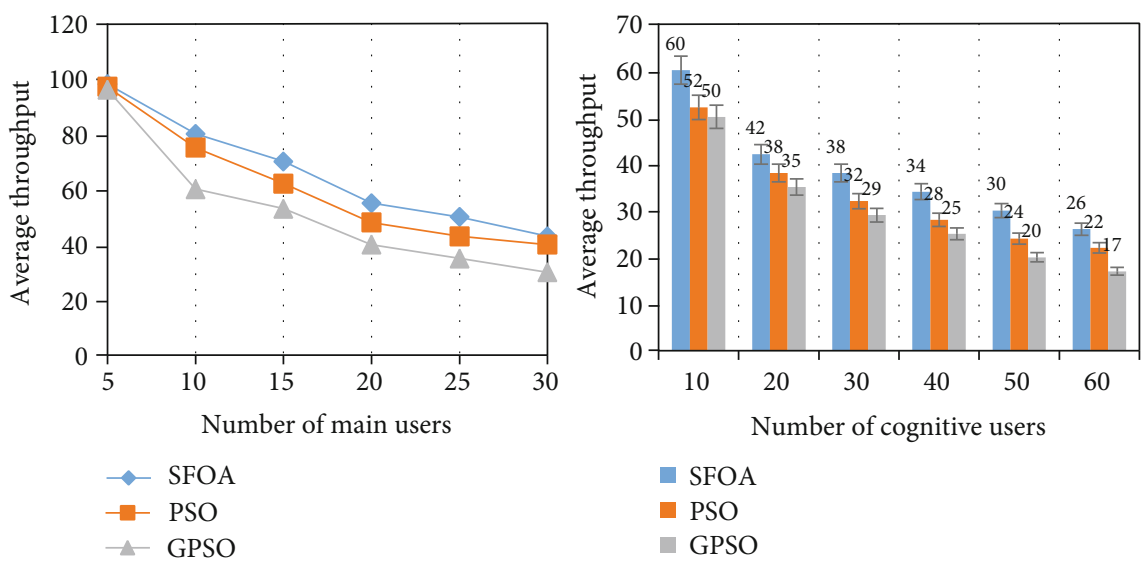

FIGURE 6: The curve of spectrum allocation performance as users increase.

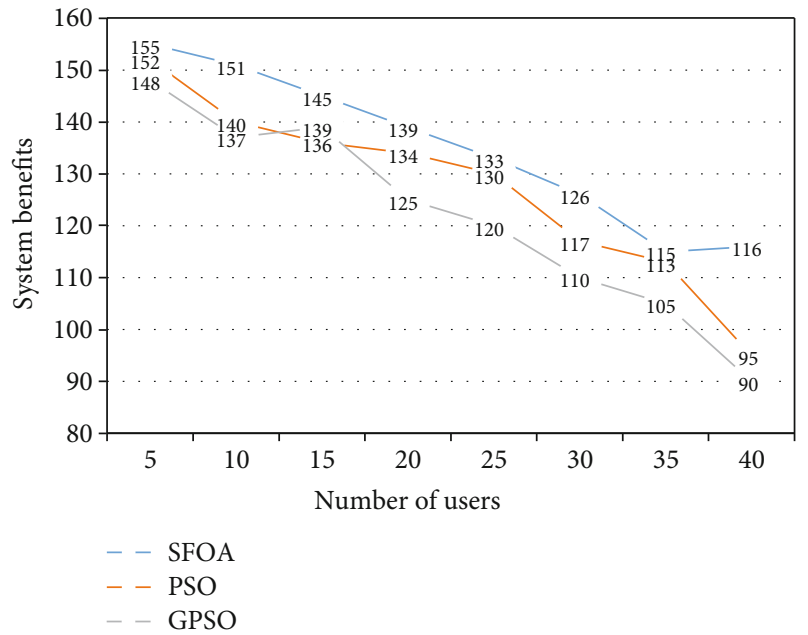

FIGURE 7: System benefits when the number of users changes.

TABLE 5: Net loss comparison.

\begin{tabular}{lccc}
\hline & SFOA & PSO & GPSO \\
\hline Average net loss & 0.1248 & 0.1287 & 0.1294 \\
Difference with initial net loss & 0.0143 & 0.0139 & 0.0134 \\
Standard deviation & 0.0019 & 0.0021 & 0.0029 \\
Reducing percentage & $11.51 \%$ & $10.52 \%$ & $9.32 \%$ \\
\hline
\end{tabular}

communication can be realized between the mobile terminal and the wireless network. The consistency of the receiving nodes of the two is relatively high, so that users can obtain satisfactory information about the Internet of Things and use this mobile computing to achieve various operations.

Currently, wireless network communication technology is highly developed, and the integration and application of technologies such as RFID in the Internet of Things to mobile terminals have become a development trend. Semantic mobile computing provides the possibility for this trend. Due to the characteristics of RFID technology, the information in wireless network communication can guarantee its own security. At the same time, we also need to pay attention to information security issues that may occur in infor- mation transmission to ensure the normal transmission of information and the user's information security. Through mobile computing, various businesses and activities in the Internet of Things can be effectively carried out, providing a good environment for its stable operation and making the information communication in the Internet of Things more reliable and effective.

Not only the mobile communication system, but also in the Internet of Things, a management and maintenance platform is also needed to realize the management and maintenance of the Internet of Things, so as to ensure that the information itself and the process of transmission remain safe and reliable. Therefore, the relevant organizational structure and the working mechanism can be applied to the Internet of Things. Due to the wide application of the Internet of Things technology, it can use its own sensor technology and RFID tags to effectively control various information. Therefore, the combination of wireless network communication technology and Internet of Things technology, plus semantic mobile computing, can effectively promote information and the rapid development of technology.

\section{Conclusion}

Starting from the spectrum allocation and optimization of wireless networks, this paper summarizes and draws on the research results of some scholars, comprehensively introduces and studies concepts such as semantic mobile computing, spectrum allocation, and optimization, and proposes the use of SFOA algorithms to solve wireless network spectrum, the method of allocation and optimization, and in-depth analysis of this algorithm and multiple algorithms in terms of performance, operating speed, throughput, and system benefits. Due to the high stability and accuracy of SFOA, through reasonable adjustment and improvement, it can make good use of spectrum allocation and optimization, which is of great significance to the development of my country's wireless communication network and the development of Internet of Things technology. This article also has some shortcomings, such as the following: it did not further improve the SFOA algorithm theoretically, but only compared it with the other two algorithms in 
several aspects; the indicators selected in the analysis part are relatively simple, without considering the impossibility. Control factors: for the feature that the SFOA algorithm can converge to the global optimal solution, there is a lack of theoretical derivation in mathematics. In response to these problems, it will be further improved in the following research to strengthen the knowledge and understanding of this field and efforts to achieve reasonable optimization and allocation of spectrum resources.

\section{Data Availability}

The data that support the findings of this study are available from the corresponding author upon reasonable request.

\section{Conflicts of Interest}

The authors declare that they have no conflicts of interest.

\section{References}

[1] E. Bertini, T. Catarci, A. J. Dix, S. Gabrielli, and G. Santucci, "Appropriating heuristic evaluation methods for mobile computing," International Journal of Mobile Human Computer Interaction, vol. 1, no. 1, pp. 20-41, 2017.

[2] K. Ma, P. Liu, J. Yang, X. Wei, and C. Dou, "Spectrum allocation and power optimization for demand-side cooperative and cognitive communications in smart grid," IEEE Transactions on Industrial Informatics, vol. 15, no. 3, pp. 1830-1839, 2019.

[3] B. Han, J. Li, J. Su, and J. Cao, "Self-supported cooperative networking for emergency services in multi-hop wireless networks," IEEE Journal on Selected Areas in Communications, vol. 30, no. 2, pp. 450-457, 2012.

[4] M. M. Sohul, M. Yao, X. Ma, E. Y. Imana, V. Marojevic, and J. H. Reed, "Next generation public safety networks: a spectrum sharing approach," IEEE Communications Magazine, vol. 54, no. 3, pp. 30-36, 2016.

[5] I. Butun, P. Österberg, and H. Song, "Security of the Internet of Things: vulnerabilities, attacks, and countermeasures," IEEE Communications Surveys \& Tutorials, vol. 22, no. 1, pp. 616644, 2020.

[6] E. Baccarelli, N. Cordeschi, A. Mei, M. Panella, M. Shojafar, and J. Stefa, "Energy-efficient dynamic traffic offloading and reconfiguration of networked data centers for big data stream mobile computing: review, challenges, and a case study," Computers \& Chemical Engineering, vol. 30, no. 2, pp. 54-61, 2016.

[7] M. R. Hossen, M. M. Rahman, K. T. Ahmed, and M. S. Miah, "Spectrum allocation management in cognitive femtocell networks for $5 \mathrm{G}$ wireless communication," Iosr Journal of Electronics \& Communication Engineering, vol. 11, no. 6, pp. 1423,2016

[8] S. Lee and R. Zhang, "Cognitive wireless powered network: spectrum sharing models and throughput maximization," IEEE Transactions on Cognitive Communications \& Networking, vol. 1, no. 3, pp. 335-346, 2015.

[9] T. Haustein, S. Stanczak, A. Wolisz et al., "Cognitive wireless communications - a paradigm shift in dealing with radio resources as a prerequisite for the wireless network of the future - an overview on the topic of cognitive wireless technologies," Frequenz, vol. 70, no. 7-8, pp. 281-288, 2016.
[10] Z. H. Yuan, C. Chen, X. Cheng, G. C. Lv, L. Q. Yang, and Y. Jin, "Correlated channel model-based secure communications in dual-hop wireless communication networks," Frontiers of Information Technology \& Electronic Engineering, vol. 18, no. 6, pp. 796-807, 2017.

[11] F. Yao, H. Wu, Y. Chen, Y. Liu, and T. Liang, "Cluster-based collaborative spectrum sensing for energy harvesting cognitive wireless communication network," IEEE Access, vol. 5, no. 99, pp. 9266-9276, 2017.

[12] L. Jun, "A synthetic research on the multimedia data encryption based mobile computing security enhancement model and multi-channel mobile human computer interaction framework," Multimedia Tools and Applications, vol. 76, no. 16, pp. 16963-16987, 2017.

[13] A. Imran, V. Quimno, and M. Hussain, "Current landscape and potential of mobile computing research in the least developed countries," The Electronic Journal of Information Systems in Developing Countries, vol. 74, no. 1, pp. 1-25, 2016.

[14] Y. Shu, K. G. Shin, H. Tian, Y. Zhang, and Y. Ye, "Proceedings of the annual international conference on mobile computing and networking, MOBICOM," Wireless Networks, vol. 3, no. 5, pp. 512-524, 2016.

[15] L. Ying-Fen, "Numerical analysis of optimization of scheduling based on fisher fishing algorithm," International Journal of Hybrid Information Technology, vol. 9, no. 6, pp. 245-252, 2016.

[16] Y. Zhang, Y. Shen, H. Wang, Y. Zhang, and X. Jiang, "On secure wireless communications for service oriented computing," IEEE Transactions on Services Computing, vol. 11, no. 2, pp. 318-328, 2018.

[17] H. S. Dhillon, H. Huang, and H. Viswanathan, "Wide-area wireless communication challenges for the Internet of Things," IEEE Communications Magazine, vol. 55, no. 2, pp. 168-174, 2017.

[18] W. Hong, Z. H. Jiang, C. Yu et al., "Multibeam antenna technologies for $5 \mathrm{G}$ wireless communications," IEEE Transactions on Antennas \& Propagation, vol. 65, no. 12, pp. 6231-6249, 2017.

[19] L. Kong, M. K. Khan, F. Wu, G. Chen, and P. Zeng, "Millimeter-wave wireless communications for IoT-cloud supported autonomous vehicles: overview, design, and challenges," IEEE Communications Magazine, vol. 55, no. 1, pp. 62-68, 2017.

[20] A. Ghazal, Y. Yuan, C. X. Wang et al., “A non-stationary IMTadvanced MIMO channel model for high-mobility wireless communication systems," IEEE Transactions on Wireless Communications, vol. 16, no. 4, pp. 2057-2068, 2017.

[21] J. M. Romero-Jerez and F. J. Lopez-Martinez, "A new framework for the performance analysis of wireless communications under Hoyt (Nakagami-q) fading," IEEE Transactions on Information Theory, vol. 63, no. 3, pp. 1693-1702, 2017.

[22] G. I. Tsiropoulos, O. A. Dobre, M. H. Ahmed, and K. E. Baddour, "Radio resource allocation techniques for efficient spectrum access in cognitive radio networks," IEEE Communications Surveys \& Tutorials, vol. 18, no. 1, pp. 824847, 2016.

[23] Y. Liu, L. Lu, G. Y. Li, Q. Cui, and W. Han, "Joint user association and spectrum allocation for small cell networks with wireless backhauls," IEEE Wireless Communications Letters, vol. 5, no. 5, pp. 496-499, 2016.

[24] M. K. Hasan, A. F. Ismail, S. Islam, W. Hashim, and B. Pandey, "Dynamic spectrum allocation scheme for heterogeneous 
network," Wireless Personal Communications, vol. 95, no. 2, pp. 299-315, 2017.

[25] A. F. Ismail, M. K. Hasan, N. I. Othman, and W. Hashim, "Assessment of dynamic spectrum allocation technique in heterogeneous network," International Journal of Future Generation Communication and Networking, vol. 10, no. 3, pp. 41-48, 2017.

[26] Y. Xiong, X. Fan, and S. Liu, "Fairness enhanced dynamic routing and spectrum allocation in elastic optical networks," IET Communications, vol. 10, no. 9, pp. 1012-1020, 2016.

[27] F. Li, K. Y. Lam, M. Jia, K. Zhao, X. Li, and L. Wang, "Spectrum optimization for satellite communication systems with heterogeneous user preferences," IEEE Systems Journal, vol. 14, no. 2, pp. 2187-2191, 2020.

[28] C. M. Baronio and A. Barth, "Correction to "The Amide I Spectrum of Proteins Optimization of Transition Dipole Coupling Parameters Using Density Functional Theory Calculations"," The Journal of Physical Chemistry B, vol. 124, no. 13, pp. 2730-2730, 2020. 